\title{
Towards a quantitative prediction of the blob detection rate
}

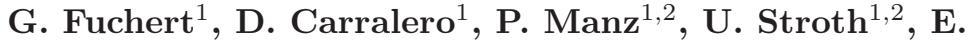 \\ Wolfrum $^{1}$, ASDEX Upgrade Team \\ ${ }^{1}$ Max-Planck-Institut für Plasmaphysik, 85748 Garching, \\ Germany \\ 2 Physik-Department E28, Technische Universität \\ München, James-Franck-Str. 1, 85748 Garching, Germany \\ E-mail: golo.fuchert@ipp.mpg.de
}

\begin{abstract}
To estimate the particle and energy flux to the plasma facing components of a future fusion power plant, the transport carried by filaments of increased pressure, called blobs, is of critical importance. To understand this transport the rate of the filaments has to be known. The novel approach presented here allows the prediction of both the blob generation and detection rates based on background plasma parameters only. The prediction is in a good overall agreement with recent experiments in ASDEX Upgrade.
\end{abstract}

PACS numbers: $52.25 . \mathrm{Xz}, 52.35 . \mathrm{Qz}, 52.35 . \mathrm{Ra}, 52.55 . \mathrm{Fa}$ 


\section{Introduction}

Filaments of increased pressure ("blobs") that transport particles and energy towards the wall are commonly observed in the scrape-off layer (SOL) of magnetically confined fusion experiments in practically all operational regimes $[1,2]$. These filaments lead to an enhanced perpendicular energy and particle transport, wall deterioration, and increased impurity densities in the edge plasma. Although many questions still remain open, especially concerning the influence of complex magnetic geometry, a lot of progress has been made in understanding the dynamics of single blobs as shown in the extensive review papers [1,2]. However, there is still no model to predict the rate of blobs, which would be of major interest for understanding and modeling the SOL physics and to predict the safe and reliable operation of future fusion power plants.

The radial fluxes due to blobs can be substantial [3]. Limiting the heat flux to plasma facing components, however, is one of the key issues in realizing fusion power plants and influencing the blob generation rate may be an important control parameter to limit the heat flux to the walls or reduce the heat flux to the divertor by increasing losses induced by filaments.

It is essential to distinguish the blob generation rate $f_{\mathrm{g}}$ from the blob detection rate $f_{\mathrm{d}}$. The definition of the latter one is straight-forward: In a pressure-sensitive signal, $f_{\mathrm{d}}$ is the number (per unit time) of large amplitude peaks exceeding a defined threshold value in a specific time interval. Knowledge of this rate is particularly important to model the plasma-wall interaction, since the large amplitude events carry the most energy and particles. Furthermore, the detection rate is the quantity accessible in experiments. Rather than being a periodic process, however, the blob ejection is intermittent and its rate can only be understood as characteristic average value. Typical rates found in the literature are e.g. around $20 \mathrm{kHz}$ for Alcator CMod [4], $4 \mathrm{kHz}$ for ASDEX Upgrade [5], $3 \mathrm{kHz}$ for DIII-D [6] and $20 \mathrm{kHz}$ for Tore Supra [7]. However, as the detection rate depends on the applied diagnostics and detection algorithm, values reported from different experiments cannot be compared directly [2] and it would be favorable to introduce a microscopic generation rate from which the experimental detection rate could be inferred. The definition of the generation rate is less obvious. Background fluctuations and merging blob events render the extraction of the exact number of single structures contributing to the signal impossible.

In this novel approach, the amplitude distribution function of the blobs relates the experimentally accessible detection rate to the generation rate, which is constrained by the dispersion of the underlying turbulence and the stability of the blobs themselves. Predictions from this model are compared to experimental values from ASDEX Upgrade.

\section{Prediction of the blob generation and detection rate}

It is a common observation (examples below) that blobs are not generated uniformly across the extent of the SOL. Instead, there is a specific radial position where the turbulence characteristics change (e.g. the cross phase between density and plasma potential, the PDF or skewness and kurtosis [8]). Radially outside of this position blobs are observed, which propagate radially outwards. Inside of the generation region, usually a micro instability is present, which does not feature a comparable radial motion. In fusion plasmas this region is observed close to the separatrix. 
However, blob generation is also observed in experiments without a confinement region. In basic plasma experiments, drift waves [9] or interchange modes [10] were observed inside of the generation region. Probe measurements in L-mode plasmas of ASDEX Upgrade indicate that the plasma turbulence close to the separatrix is dominated by a drift-wave-like instability [11, 12]. Waiting-time analyses from the linear device PISCES [13] and the low-temperature stellarator TJ-K [14] revealed a close coupling between coherent structures inside and outside of the generation region. A similar observation is reported for LHD [15] together with experimental evidence for a link between the waiting-time statistics and the instability drive (global $\beta$ and edge pressure gradient) [16]. The detachment of blobs from coherent structures has even been visualized directly in basic plasma experiments like TJ-K [9] and the simple magnetized torus TORPEX [10], but also in fusion experiments like Alcator C-Mod [17], NSTX [18], and TEXTOR [19].

All of these observations point to the fact that a dominant edge instability (not necessarily the same for all experiments) is involved in the blob generation process as source for seed fluctuations, i. e. structures which locally increase the pressure gradient and thereby trigger blob generation. Even non-local effects like turbulence spreading can act as source for seed fluctuations [20, 21].

Hence, it is assumed in the presented phenomenological model that the generation rate is given by the frequency of this dominant edge instability. Since the frequency is linked to the spatial structure size by the dispersion relation of that instability it is necessary to know on which size scale the blob generation takes place. There is experimental evidence that the characteristic size (perpendicular to the magnetic field) of coherent structures in fusion plasmas remains either constant when crossing the generation region, as observed in ASDEX Upgrade [8], or changes slowly and continuously without jumps (e.g. at JET [22] or DIII-D [23]). This implies that blobs are generated by coherent structures of comparable size and that the blob size close to the generation region can be used as a proxy for the scale of the generating instability. Another way to look at this is that there exists an intermediate blob size $\delta_{\mathrm{b}}$, where coherent blobs can form [24] and are most stable against secondary instabilities (Kelvin-Helmholtz and interchange instability) [25, 26]. Therefore, the most stable blob size defines a selection rule on which scale the seed fluctuations can develop to blobs which are stable enough to propagate over a larger distance.

For a fixed observer the characteristic occurrence rate of seed fluctuations is given by the (poloidal) background $\mathbf{E} \times \mathbf{B}$-drift velocity $v_{\mathrm{E}_{r} \times \mathrm{B}}$ and the phase velocity $v_{\mathrm{ph}}$ of the instability:

$$
f_{\mathrm{g}}=\left(v_{\mathrm{ph}}+v_{\mathrm{E}_{r} \times \mathrm{B}}\right) / \lambda_{\perp} .
$$

This equation predicts the local blob generation rate in regions of active blob drive in the most general form of the presented phenomenological model. In this representation the model can be regarded as modular in the sense that it generalizes about the type of instability triggering blob generation and the relevant blob dynamics. Although some of the experiments mentioned above suggest such a linear relationship between the frequency of seed fluctuations and the number of generated blobs, at this point the linearity has to be assumed. Dedicated experiments and simulations are required to gain further insight into this important aspect of blob generation.

In order to predict actual generation rates for a given experiment, the seed instability has to be identified together with a prediction of the radial electrical field at the separatrix. For ASDEX Upgrade L-mode discharges it is assumed that in 
the SOL $E_{r}=-2.4 \nabla T_{\mathrm{e}} / e$ (estimated from the relationship between floating and plasma potential in the SOL, see e.g. Ref. [27], assuming deuterium plasmas and $\left.T_{i} / T_{e} \approx 3[28]\right)$ and, as discussed above, that the seed instability is drift-wave-like. Hence, $v_{\mathrm{ph}}$ is approximated by the diamagnetic velocity $v_{\text {dia }}$. Note that the model relies on the existence and instability of such a generating micro-instability in the generation region, which it cannot predict itself. The identification of the type of the generating mode and assessment of its stability for given plasma conditions and field geometries are out of the scope of this phenomenological model and require external input. As discussed above, for typical ASDEX Upgrade plasmas, a drift-wave-like instability in the vicinity of the separatrix is assumed. It follows that

$$
f_{\mathrm{g}}=\left|\frac{1}{3 \delta_{\theta}} \cdot\left(\frac{\nabla p_{\mathrm{e}}}{e n_{\mathrm{e}} B}-2.4 \frac{\nabla T_{\mathrm{e}}}{e B}\right)\right|,
$$

with the electron density $n_{\mathrm{e}}$, pressure $p_{\mathrm{e}}$ and temperature $T_{\mathrm{e}}$, the elementary charge $e$ and magnetic field strength $B$. Here, the conversion between the poloidal blob size $\delta_{\theta}$, defined as the full (poloidal) width at half maximum (FWHM) of the density perturbation, and the poloidal wave length $\lambda_{\perp}$ of the dominant edge instability $\lambda_{\perp}=3 \delta_{\theta}$ has been used, since the FWHM of the positive part of a sine wave is approximately $\lambda / 3$. This can be regarded as a modification of the commonly invoked blob correspondence principle [4]. Note that this relation is chosen due to the experimental observations discussed above that blobs are generated by density perturbations of comparable size.

It should be noted that due to the turbulence in fusion plasmas the generation is not induced by a stable, coherent mode, but rather by transient quasi-coherent fluctuations that are part of the turbulent mode spectrum. In this case, blob generation is not strictly periodic and the presented model predicts a statistical average frequency.

Due to the small fraction of large amplitude events and the experimental focus on these, most of the generated structures are not detected. The detection rate $f_{\mathrm{d}}$ can be predicted as follows: The seed instability generates blobs at a rate $f_{\mathrm{g}}$. They propagate radially outward, where a fraction $\gamma_{\mathrm{d}}$ of them is detected, depending on the blob amplitude distribution function and threshold amplitude $a_{\text {thresh }}$ :

$$
f_{\mathrm{d}}=f_{\mathrm{g}} \gamma_{\mathrm{d}} \text {. }
$$

For a given amplitude distribution, $\gamma_{\mathrm{d}}$ can be estimated by

$$
\gamma_{\mathrm{d}}=1-\frac{\left.\mathrm{CDF}\right|_{0} ^{a_{\text {thresh }}}}{\left.\mathrm{CDF}\right|_{0} ^{\infty}},
$$

where $\left.\mathrm{CDF}\right|_{a} ^{b}$ (cumulative distribution function) denotes the integral of the distribution from $a$ to $b$. Since the amplitude distribution function in the generation region is not known, $\gamma_{\mathrm{d}}$ will be determined from experimental data below.

This line of argument is only valid as long as the blob transit time at a given position $\tau_{\mathrm{d}}$ is small compared to the characteristic waiting time $\tau_{\mathrm{w}}=1 / f_{g}$. In a simple model, for $\tau_{\mathrm{d}} \geq 1.7 \tau_{\mathrm{w}}$, a typical blob is a compilation of $\left(\tau_{\mathrm{d}} / \tau_{\mathrm{w}}\right)+0.3$ single events (see Fig. 1 and its discussion in the Appendix). This reduces the number of clearly distinguishable blobs. In this case the detection rate $f_{d}^{*}$ (to distinguish it from $f_{\mathrm{d}}$ from Eq. $\left.(3)\right)$ is predicted as

$$
f_{\mathrm{d}}^{*}=\frac{f_{\mathrm{g}}}{\tau_{\mathrm{d}} / \tau_{\mathrm{w}}+0.3} \gamma_{\mathrm{d}}=\frac{1}{\tau_{\mathrm{d}}+0.3 \tau_{\mathrm{w}}} \gamma_{\mathrm{d}} .
$$




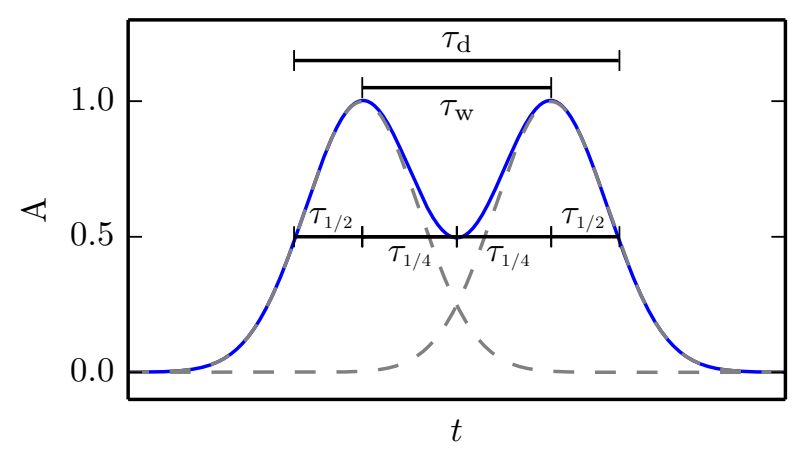

Figure 1. In a time series of a pressure sensitive quantity $A$, single blobs are not properly resolved if the blob transit time $\tau_{\mathrm{d}} \geq 1.7 \tau_{\mathrm{w}}$. A characteristic structure (closed line) is a compilation of $\left(\tau_{\mathrm{d}} / \tau_{\mathrm{w}}\right)+0.3$ single events (dashed lines). The derivation of these relations is shown in the Appendix. $\tau_{1 / 2}$ is the full duration at half maximum and $\tau_{1 / 4}$ the full duration at quarter maximum.

In principle, due to the poloidal and radial motion of the blobs it would be possible that the typical blob trajectory (depending on these two velocity components) would have to be taken into account when calculating the detection rate at a fixed location. However, as long as the generation rate changes only slowly in the poloidal direction, the propagation of blobs has only a minor impact on the detection rate, since on average the characteristic time to reach the observation region is constant and does not influence the time distance between consecutive events.

For the following comparison with experimental results it is assumed that the detection rate $f_{\mathrm{d}}$ is dominated by single structure effects, Eq. (3), for $\tau_{\mathrm{d}} / \tau_{\mathrm{w}}<1.7$ and by Eq. (5) otherwise. Furthermore, a circular blob shape is assumed, i. e. the radial size is equal to the poloidal one $\left(\delta_{r}=\delta_{\theta}\right)$. This assumption is only employed to estimate the blob transit time $\tau_{\mathrm{d}}$ to assess the role of blob merging on the detection rate. Simulations show (see e.g. [29, 30]) that depending on the SOL plasma conditions, blobs feature different shapes with either $\delta_{r} \approx \delta_{\theta}$ (circular) or $\delta_{r}<\delta_{\theta}$. In the latter case, the assumption of a circular blob shape obviously does not hold and $\tau_{\mathrm{d}}$, and hence the impact of blob merging, would be overestimated. In the ASDEX Upgrade results presented below, blob merging is only of minor importance both for measured and predicted values of $\tau_{\mathrm{d}}$, which justifies the simple assumption of a circular blob shape. Experiments usually show no large difference between $\delta_{r}$ and $\delta_{\theta}$. For example in NSTX it is observed that over almost the whole operational range the ratio of $\delta_{r}$ and $\delta_{\theta}$ varies only between 0.5 and 2.0 [31].

\section{Comparison with experimental data}

Blob detection rates were measured in a series of L-mode discharges at ASDEX Upgrade (\#29321-29326 and \#29887, all in 2013). In these experiments, blob properties were studied using a 14-pin probe. Cross-correlation based analysis techniques were used to determine the blob size, velocity, and transit time. The 
detection rate was determined by a peak detection algorithm. Detailed studies on blob dynamics in these discharges were already presented in Ref. [3], where the main focus lies on identifying the relevant dependences of the blob properties on the background plasma parameters and studying a possible feedback between the two. Since the same experimental data is used in the following, the details regarding the probe geometry, data analysis and blob dynamics in these discharges are given in Ref. [3].

While the magnetic parameters and heating power were kept constant in these experiments (toroidal magnetic field $B_{\mathrm{t}}=-2.5 \mathrm{~T}$, safety factor $q_{95}=5.32$, plasma current $I_{\mathrm{p}}=800 \mathrm{kA}$, and ECRH power of $\left.600 \mathrm{~kW}\right)$, the fueling level was varied considerably to achieve line integrated edge densities $\bar{n}_{\mathrm{e}, \mathrm{H}-5}$ (see e.g. Ref. [3]) between approximately $8 \cdot 10^{18}$ and $3.5 \cdot 10^{19} \mathrm{~m}^{-2}$. In this range a significant change in the blob dynamics is observed at ASDEX Upgrade as will be discussed below.

Using Langmuir probes, the blob detection rate $f_{\mathrm{d}}$ was measured for different edge plasma parameters. The kinetic profiles (electron temperature and density) required for evaluating the model equations are deduced from the standard edge diagnostics at ASDEX Upgrade [32].

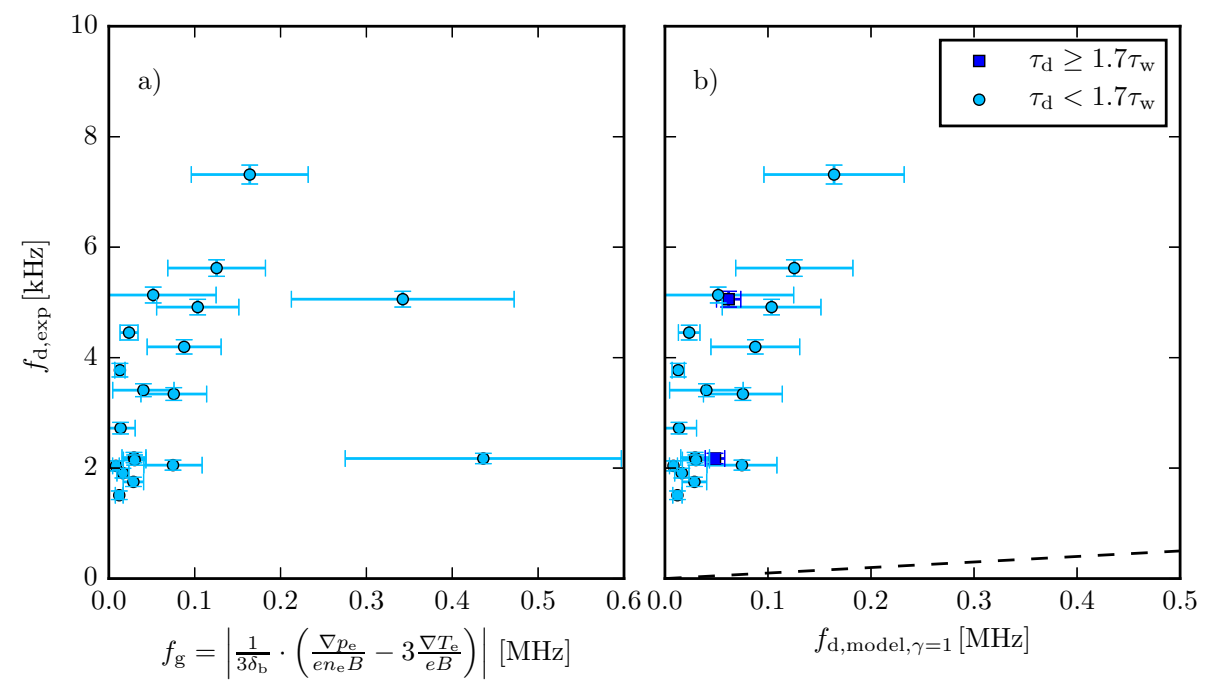

Figure 2. Blob detection rate $f_{\text {d,exp }}$ from probe data around $\rho_{\text {pol }}=1.025$ plotted over a) the generation rate $f_{\mathrm{g}}$ calculated from Eq. (2) evaluated at the separatrix and b) the detection rate according to Eq. (3) or (5), depending on the factor $\tau_{\mathrm{d}} / \tau_{\mathrm{w}}$, for $\gamma_{\mathrm{d}}=1$. Note the different scale of the axes, the dashed line would indicate a perfect match of the prediction with the experiment.

Figure 2 a) shows the measured detection rate $f_{\text {d,exp }}$ as a function of the predicted generation rate $f_{\mathrm{g}}$, according to Eq. (2) evaluated at the separatrix (which at the same time yields $\left.\tau_{\mathrm{w}}=1 / f_{\mathrm{g}}\right)$. From the discussion above it is clear that $f_{\mathrm{g}} \neq f_{\mathrm{d}}$. Nevertheless, an overall trend can be observed that the detection rate increases with the generation rate with two clear outliers above a predicted generation rate $f_{\mathrm{g}} \approx 300 \mathrm{kHz}$. In Fig. $2 \mathrm{~b}$ ) the prediction for the detection rate $f_{\mathrm{d}}$ is shown for the unrealistic case that there are no events with amplitudes below the detection threshold $\left(\gamma_{\mathrm{d}}=1\right)$. In the graph the circles correspond to the predictions of Eq. (3) for $\tau_{\mathrm{d}} / \tau_{\mathrm{w}}<1.7$ and the squares to $\tau_{\mathrm{d}} / \tau_{\mathrm{w}} \geq 1.7$, Eq. (5). It can be seen that due to the correction for merging events the outliers seen in Figure 2 a) now fall within the 
group of the other data.

From these data and Eqs. (3) and (5), the unknown $\gamma_{\mathrm{d}}$ can be estimated. For the evaluation, $\tau_{\mathrm{d}}$ is identified with the radial auto-correlation time of the probe signal. A good agreement is obtained for $\gamma_{\mathrm{d}}=0.065$, as is shown in Fig. 3, where again the circles correspond to the predictions of Eq. (3) for $\tau_{\mathrm{d}} / \tau_{\mathrm{w}}<1.7$ and the squares to $\tau_{\mathrm{d}} / \tau_{\mathrm{w}} \geq 1.7$, Eq. (5). It can be seen that the observed detection rate scales linearly with the model values, however, the slope is smaller than predicted. This may point to a more complex blob shape and amplitude distribution, which both may even vary depending on the edge plasma parameters. Furthermore, assuming the ideal driftwave dispersion relation for the dominant edge instability and the constancy of the blob size from the generation to the detection region may have to be refined and should be studied in more detail in dedicated experiments. Nevertheless, for the majority of points the absolute values are predicted correctly within a factor of two. Hence, the phenomenological picture of blob generation by seed fluctuations on the same size scale is indeed capable to quantitatively understand experimental blob detection rates.

The experimental value of $\gamma_{\mathrm{d}}=0.065$ can be compared to theoretical predictions evaluating Eq. (4) with $a_{\text {thresh }}=2.5 \sigma$ ( $\sigma$ being the standard deviation of the fluctuation signal) for a Gaussian distribution, $\gamma_{\mathrm{d}}^{\text {norm }} \approx 0.0124$, and an exponential distribution (recently discussed in Ref. [33]), $\gamma_{\mathrm{d}}^{\exp } \approx 0.0821$. It can be seen that the probability for larger amplitudes is increased compared to a Gaussian distribution, but remains smaller than for an exponential distribution $\left(\gamma_{d}^{\text {norm }}<\gamma_{d}<\gamma_{d}^{\text {exp }}\right)$.

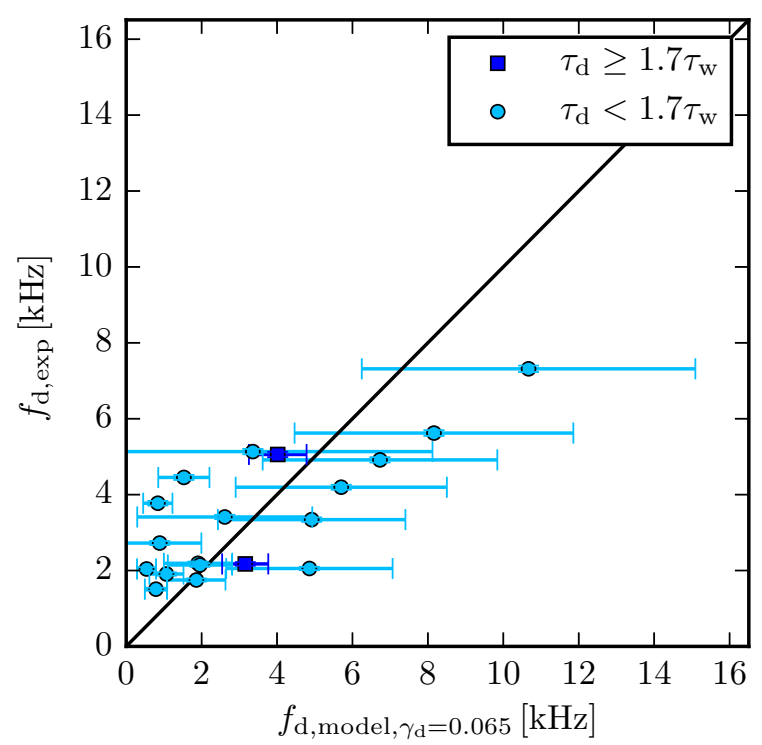

Figure 3. Experimental blob detection rate $f_{\text {d,exp }}$ around $\rho_{\text {pol }}=1.025$ compared to a prediction from Eq. (3) for $\tau_{\mathrm{d}}<1.7 \tau_{\mathrm{w}}$ and Eq. (5) for $\tau_{\mathrm{d}} \geq 1.7 \tau_{\mathrm{w}}$, respectively for $\gamma_{\mathrm{d}}=0.065$.

The successful prediction of $f_{\mathrm{d}}$ included experimental data for $\delta_{\theta}$ and $\tau_{\mathrm{d}}$ from probe measurements. This is already an important step towards a quantitative understanding of the blob generation process. A predictive model, however, should preferably only depend on either measured or simulated density and temperature 
profiles. Now, the model equations can be evaluated by taking the most stable blob size $\delta_{\mathrm{b}}$ as the poloidal blob size and estimating $\tau_{\mathrm{d}} \approx \delta_{\mathrm{b}} / v_{\mathrm{b}, \mathrm{r}}$ (at this point the assumption of a circular blob shape is used). A lot of effort has been put on finding and validating analytical models for $\delta_{\mathrm{b}}$ and $v_{\mathrm{b}, \mathrm{r}}$ (see e. g. Ref. [2] and references therein).

At ASDEX Upgrade, the identification of the relevant blob regimes is subject of present research $[5,34,35]$. One of the important results is that there are at least two different regimes relevant for the typical ASDEX Upgrade L-mode discharges depending on the connection of the plasma to the target plates. For low edge densities the size and velocity of blobs seems to scale as predicted by the well-studied sheath limited regime [36], probably influenced by a finite ion temperature $T_{\mathrm{i}}[37]$.

Following Ref. [37] and assuming that in the SOL $T_{\mathrm{i}} \approx 3 T_{\mathrm{e}}[28], l_{\|}=70 \mathrm{~m}$ (parallel connection length from field line tracing) and $R=2.17 \mathrm{~m}$ (curvature radius at the separatrix), the following scaling laws for blob size and radial velocity are obtained:

$$
\delta_{b}=4 \rho_{\mathrm{s}}\left(\frac{l_{\|}^{2}}{\rho_{\mathrm{s}} R}\right)^{1 / 5}, \quad v_{\mathrm{b}, r}=4 c_{\mathrm{s}}\left(\frac{\rho_{\mathrm{s}}}{\delta_{\mathrm{b}} / 2}\right)^{2} \frac{l_{\|}}{R} .
$$

Note that the theoretical scalings usually define $\delta_{\mathrm{b}}$ (in contrast to the definition in this paper) as half width at half maximum. This is accounted for by replacing $\delta_{\mathrm{b}}$ in the scalings by $\delta_{\mathrm{b}} / 2$.

As is described in Ref. [35], the blob dynamics in ASDEX Upgrade is changing from the sheath limited regime described above to the inertial regime $[3,35,38]$ when the effective collisionality parameter $\Lambda[3,39]$ is larger than approximately 1 (high collisionality). In the data set used here this is the case above a threshold lineintegrated edge density of $\bar{n}_{\mathrm{e}, \mathrm{H}-5} \approx 2.5 \cdot 10^{19} \mathrm{~m}^{-2}$. In ASDEX Upgrade, the radial velocity in this regime scales like [35]

$$
v_{\mathrm{b}, r}=\frac{c_{\mathrm{s}}}{2} \sqrt{0.33 \delta_{\mathrm{b}} R}
$$

while the blob size is observed to scale linearly with the density. So far, however, there is no prediction for the blob size in the inertial regime. Therefore, the empirical scaling for the blob size

$$
\delta_{\text {inertial }}[\mathrm{m}]=0.047 \cdot\left(\bar{n}_{\mathrm{e}, \mathrm{H}-5}\left[10^{19} \mathrm{~m}^{-2}\right]\right)-0.058
$$

is assumed in accordance with the experimental values as shown in Fig. 4.

This leads to a prediction of the generation rate $f_{\mathrm{g}}$ in the sheath limited regime of

$$
f_{\mathrm{g}}=\left|\frac{1}{12 \rho_{\mathrm{s}}} \cdot\left(\frac{\rho_{\mathrm{s}} R}{l_{\|}^{2}}\right)^{1 / 5} \cdot\left(\frac{\nabla p_{\mathrm{e}}}{e n_{\mathrm{e}} B}-2.4 \frac{\nabla T_{\mathrm{e}}}{e B}\right)\right|,
$$

and in the inertial regime

$$
f_{\mathrm{g}}=\left|\frac{1}{3 \cdot\left(0.047 \cdot\left(\bar{n}_{\mathrm{e}, \mathrm{H}-5}\left[10^{19} \mathrm{~m}^{-2}\right]\right)-0.058\right)} \cdot\left(\frac{\nabla p_{\mathrm{e}}}{e n_{\mathrm{e}} B}-2.4 \frac{\nabla T_{\mathrm{e}}}{e B}\right)\right|,
$$

To obtain the experimental detection rate $f_{\mathrm{d}}, \tau_{\mathrm{d}}$ has to be predicted to decide whether Eq. (3) or (5) have to be used and $\gamma_{\mathrm{d}}$ is needed for the evaluation of these equations. Using $\tau_{\mathrm{d}} \approx \delta_{\mathrm{b}} / v_{\mathrm{b}, \mathrm{r}}$, as discussed above, for the sheath limited regime it is

$$
\tau_{\mathrm{d}} \approx \frac{4}{c_{\mathrm{s}}}\left(R^{2} \rho_{\mathrm{s}}^{2} l_{\|}\right)^{1 / 5}
$$




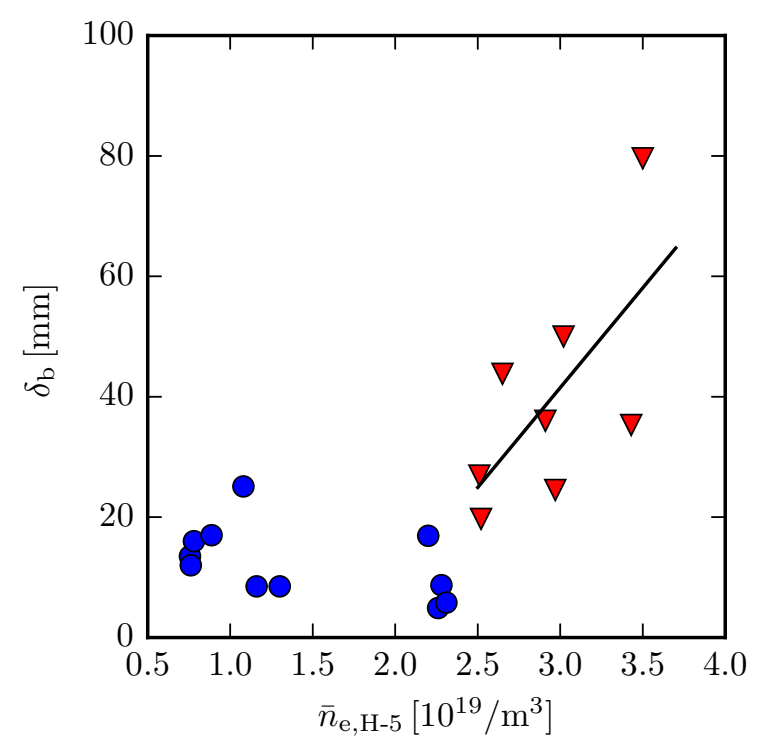

Figure 4. Blob size for the analyzed discharges plotted over the line integrated edge density. Above a threshold density a linear fit (black line) is used to describe the experimental data points (red triangles). For lower densities (blue dots) no such relation is observed.

and for the inertial regime

$$
\tau_{\mathrm{d}} \approx\left(0.047 \cdot\left(\bar{n}_{\mathrm{e}, \mathrm{H}-5}\left[10^{19} \mathrm{~m}^{-2}\right]\right)-0.058\right) \cdot \frac{2}{c_{\mathrm{s}}}
$$

Using these equations together with the experimental value for $\gamma_{\mathrm{d}}$, the prediction of $f_{\mathrm{d}}$ is repeated and the result is shown in Fig. 5. Compared to Fig. 3 the data points show a stronger scatter, mainly due to the fact that the values for $\delta_{\mathrm{b}}$ and $v_{\mathrm{b}, r}$ calculated from Eqs. (6) and (8) are not in perfect agreement with the experimental ones. The absolute values, however, are again predicted correctly within a factor of two for most data points. Furthermore, it can be seen that a higher number of discharges is predicted to have $\tau_{\mathrm{d}} / \tau_{\mathrm{w}}<1.7$.

Using the presented phenomenological model, it was possible for the first time to predict blob detection rates for ASDEX Upgrade L-mode discharges with the kinetic profiles as only input. In order to achieve this, the ratio between generated seed fluctuations and detected blobs $\gamma_{d}$ had to be obtained experimentally. However, the empirical value for ASDEX Upgrade of $\gamma_{\mathrm{d}}=0.065$ is of the same order of magnitude as the one calculated for blobs with exponentially distributed amplitudes $\left(\gamma_{d}^{\exp } \approx 0.0821\right.$, using the experimental detection threshold of $\left.a_{\text {thresh }}=2.5 \sigma\right)$, which can be used as a reasonable estimate when no experimental data is available. It is clear, however, that especially in the inertial regime a better understanding is required to better predict $\delta_{\mathrm{b}}$ and $v_{\mathrm{b}, r}$. This is of particular importance since ITER will be operating in this regime [40, 41] and probably DEMO as well [42] (depending on the final design parameters). 


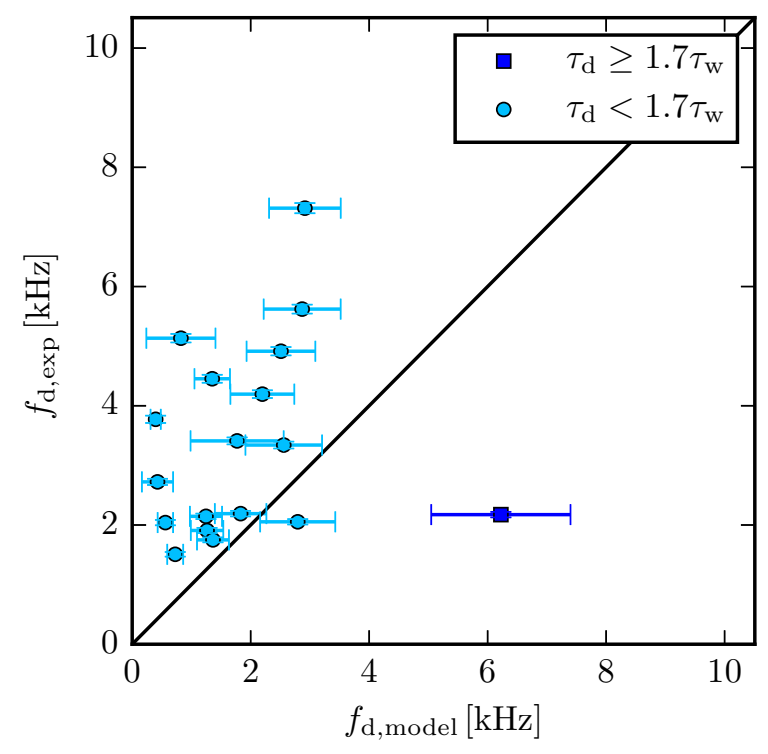

Figure 5. Same representation as in Fig. 3, but using analytical scaling laws for $\delta_{\mathrm{b}}$ and $\tau_{\mathrm{d}}$ instead of experimental data.

\section{Summary and conclusion}

From the experimental findings that blobs are generated in a distinct region close to the separatrix by a dominant edge instability and that there seems to be a relation between this instability and the blobs in terms of detection frequency and size, a phenomenological model was inferred, which predicts for the first time the blob detection rate $f_{\mathrm{d}}$ as a function of the edge kinetic profiles (electron temperature and density) by calculating the generation rate of seed fluctuations $f_{\mathrm{g}}$ and determining the ratio of generated seed fluctuations and detected blobs $\gamma_{\mathrm{d}}$. The underlying distributions of size, velocity and waiting time are represented by characteristic average quantities.

Depending on the characteristic waiting time and the blob transit time at a given position, the detected structures are either observed as individual blobs $\left(f_{\mathrm{d}}=\gamma_{\mathrm{d}} f_{\mathrm{g}}\right)$ or as compound structures (Eq. (5)). The model itself generalizes about different blob regimes and dominant edge instabilities and can therefore be adapted to different experiments and plasma conditions.

A comparison with experimental detection rates has been carried out for ASDEX Upgrade L-mode discharges. By assuming a drift-wave-like instability $\left(f_{\mathrm{g}}\right.$ given by Eq. (2)) in the generation region, a good overall agreement was achieved for $\gamma_{\mathrm{d}}=0.065$, which is close to expectations from an exponential blob amplitude distribution function. Further experiments will reveal how robust the presented results are over a broader range of plasma conditions, including variations in the toroidal field strength and different heating scenarios.

The prediction of the detection rate of plasma blobs is of great use for practical considerations about the blob transport and plasma-wall interaction, since especially the large amplitude events carry the most energy and particles. Furthermore, influencing the blob generation rate may also be an important control parameter for 
the heat flux to the divertor, since it has been shown that the radial fluxes due to blobs can be substantial: In ASDEX Upgrade high-collisionality regimes a flattening of the SOL density profile is observed and explained by a transport increase due to filamentary transport $[3,35,43]$. Similar profile changes have also been observed in other tokamaks including JET $[41,44,45]$, which indicates that the ASDEX Upgrade results probably illustrates a general phenomenon.

The presented model enables the quantitative prediction of the blob detection rate in a fusion plasma, using the kinetic profiles as only input. Altogether, this prediction is an important step towards a deeper and more quantitative understanding of the blob generation process and may offer a perspective to an active control of blob transport by profile shaping in the generation region.

\section{Acknowledgments}

This work has been carried out within the framework of the EUROfusion Consortium and has received funding from the Euratom research and training programme 20142018 under grant agreement No 633053. The views and opinions expressed herein do not necessarily reflect those of the European Commission. Furthermore, this work is supported by Agence Nationale pour la Recherche, contract ANR-11-BS09-023-03 (SEDIBA).

\section{Appendix}

In the case of small waiting times $\tau_{\mathrm{w}}$, subsequent blobs will merge before propagating out of the generation region. Here, small refers to $\tau_{\mathrm{w}}$ approaching the blob transit time $\tau_{\mathrm{d}}$. Experimentally, $\tau_{\mathrm{d}}$ is inferred from the width of the autocorrelation function of the blob detecting signal. In the presented experiments, the full width at half maximum (FWHM) of the autocorrelation function of the Langmuir probe reference pin was used as shown in Fig. 6. In contrast to the experimental values, the predicted ones, $\tau_{\mathrm{d}} \approx \delta_{\mathrm{b}} / v_{\mathrm{b}, \mathrm{r}}$, neglect the influence of the poloidal velocity. In order to make the model consistent with this assumption, throughout this paper instead of the transit time $\tau_{\mathrm{d}}$, the radial transit time $\tau_{\mathrm{d}, \mathrm{r}}=\tau_{\mathrm{d}} / \cos \alpha$ (where $\alpha$ is the angle between the measured propagation direction of the blob and the binormal direction as defined in Ref. [3]) is used.

A proper statistical approach to study the consequences of these effects requires the waiting time, size, and amplitude distributions and non-linear interactions between different blobs would have to be taken into account. This is, however, an outstanding task of blob research and outside the scope of a simple model. In order to nevertheless estimate the reduction of detected blobs due to blob interaction the distributions of the blob properties are replaced by their characteristic average values. Furthermore it is assumed that the blob shape is Gaussian and that different blobs interfere linearly. The Gaussian blob shape is used for analytical simplicity and does not describe the steep front and long tail well that are observed experimentally. It is shown below that the results obtained for a Gaussian blob shape are not changed significantly by considering a more realistic blob shape.

If a blob is experimentally defined by the amplitude structure limited by the full width at half maximum (FWHM) with respect to the detected maximum amplitude $A_{\text {max }}$, blob interaction plays a role as soon as the time distance between two blobs 


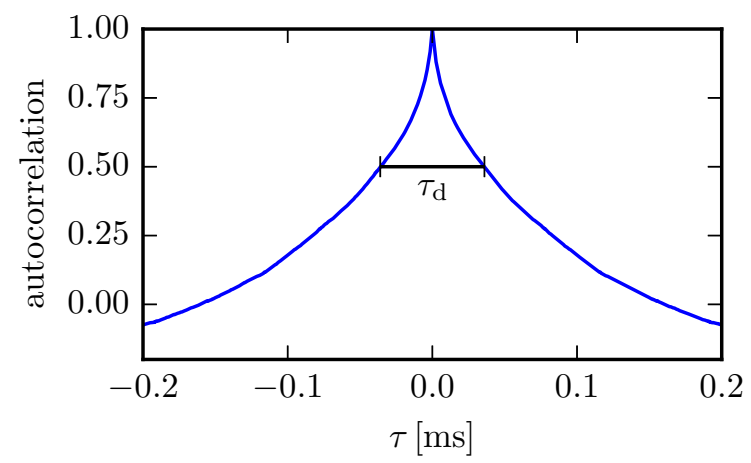

Figure 6. Autocorrelation function of the ion saturation current signal of the reference probe tip in one of the presented discharges $(\# 29321, t \approx 1.93 \mathrm{~s})$. The transit time $\tau_{\mathrm{d}}$ is defined as the FWHM of this autocorrelation function.

is short enough so that they merge at $A_{\max } / 4$. It can be calculated from Fig. 1 (for Gaussian blobs) that the minimum blob transit time $\tau_{\mathrm{d} \text {, min }}$ for this is

$$
\tau_{\mathrm{d}, \min }=2 \tau_{1 / 2}+2 \tau_{1 / 4}
$$

where $\tau_{1 / 2}$ is the full duration at half maximum and $\tau_{1 / 4}$ the full duration at quarter maximum. Furthermore, note that $\tau_{1 / 4}=\sqrt{2} \tau_{1 / 2}$ and, for the situation shown in the figure, $2 \tau_{1 / 4}=\tau_{\mathrm{w}}$. With these relations it follows that

$$
\tau_{\mathrm{d}, \min }=\left(1+\frac{1}{\sqrt{2}}\right) \tau_{\mathrm{w}} \approx 1.7 \tau_{\mathrm{w}} .
$$

Hence, according to this simple model blob interaction has to be considered for $\tau_{\mathrm{d}} \geq 1.7 \tau_{\mathrm{w}}$. It remains to be calculated how many single events contribute on average $(\bar{n})$ to one detected blob structure. From considerations similar to the one shown in Fig. 1 it can be inferred that

$$
\tau_{\mathrm{d}}=\bar{n} \tau_{\mathrm{w}}+2 \tau_{1 / 2}
$$

From the experimental blob definition it is clear that $2 \tau_{1 / 4}=2 \sqrt{2} \tau_{1 / 2} \leq \tau_{\mathrm{w}}$ and, hence, it is approximated that

$$
\tau_{\mathrm{d}} \approx(\bar{n}-1) \tau_{\mathrm{w}}+\frac{1}{\sqrt{2}} \tau_{\mathrm{w}}
$$

Finally, $\bar{n}$ can be calculated as

$$
\bar{n}=\frac{\tau_{\mathrm{d}}}{\tau_{\mathrm{w}}}+1-\frac{1}{\sqrt{2}} \approx \frac{\tau_{\mathrm{d}}}{\tau_{\mathrm{w}}}-0.3 .
$$

As mentioned above, both experiments and simulations show a radial blob density profile which deviates from a Gaussian density profile. Rather, in the direction of motion, there is a clear steepening of the density front, while on the other side the blob density profile features a longer tail. Analytically sometimes the extreme case of a Heaviside step function for the sharp rise and an exponential decay for the tail is considered to describe the blob shape (e.g. in Ref. [46]). 
An example for two overlapping blobs with such a shape is seen in Fig. 7. It should be mentioned that the displayed blob structure features amplitudes lower than $1 / 2$ (with 1 being the peak amplitude). This points to an issue with simple amplitudebased detection algorithms for blobs: The left structure at smaller time values is observed first, reaching the amplitude detection threshold. Before the amplitude falls below $1 / 2$ of this triggering value, an amplitude is recorded, which is smaller than twice the triggering value, but so large that not all amplitude values in between the two peaks are above half the peak amplitude of the second peak. Hence, the initial pulse could neither be detected as isolated single structure, nor belong to the second, larger pulse. In order to deal with this situation, many blob detection algorithms accept amplitude values within one structure below one half of the peak amplitude, if they clearly do not belong to an isolated single structure.

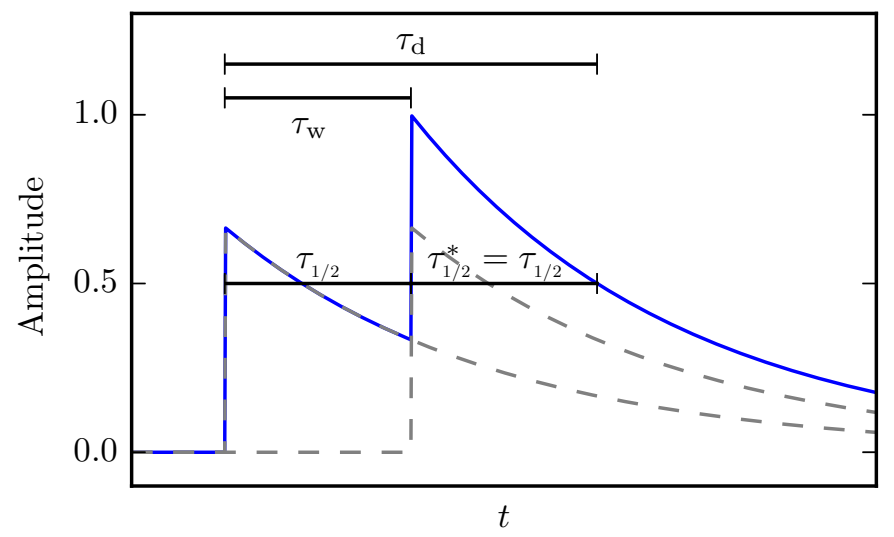

Figure 7. Time series of two linearly interacting perturbations assuming an idealized blob shape composed of a Heaviside step function followed by an exponential decay.

From Fig. 7 it can be seen that in this case

$$
\tau_{\mathrm{d}, \min }=\tau_{1 / 2}^{*}+\tau_{\mathrm{w}} .
$$

Here, $\tau_{1 / 2}^{*}$ is the half-life period of the merged pulses. For the sum of two exponential decays with identical half-life period $\tau_{1 / 2}$, however, $\tau_{1 / 2}^{*}=\tau_{1 / 2}$. For the statistical average it can be assumed that $\tau_{1 / 2} \approx \tau_{\mathrm{w}}$, since for $\tau_{1 / 2} \gg \tau_{\mathrm{w}}$, even more blobs would contribute to the merged structure and for $\tau_{1 / 2} \ll \tau_{\mathrm{w}}$ blob merging should not play a role at all. Hence it can be assumed that $\tau_{\mathrm{d}, \min }=2 \tau_{\mathrm{w}}$, which is close to the $1.7 \tau_{\mathrm{w}}$ obtained for a Gaussian blob shape.

Furthermore, it can be concluded that

$$
\tau_{\mathrm{d}}=\tau_{1 / 2}+(\bar{n}-1) \tau_{\mathrm{w}} \approx \bar{n} \tau_{\mathrm{w}}
$$

and, hence

$$
\bar{n}=\frac{\tau_{\mathrm{d}}}{\tau_{\mathrm{w}}}
$$

instead of $\tau_{\mathrm{d}} / \tau_{\mathrm{w}}-0.3$. Considering the limitations of this simple model it can be concluded that the influence of the pulse shape has no strong impact on the results. 
For the ASDEX Upgrade discharges analyzed in this paper, the experimentally observed blob shape falls somewhere in between the considered extreme cases, as has been shown in Ref. [3] using exactly the same probe data analyzed in this paper, which justifies for this purpose to assume a Gaussian blob pulse shape.

\section{References}

[1] Krasheninnikov S I, D'Ippolito D A, and Myra J R 2008 J. Plasma Phys. 74679

[2] D'Ippolito D A, Myra J R, and Zweben S J 2011 Phys. Plasmas 18060501

[3] Carralero D, Birkenmeier G, Müller H W, Manz P, de Marne P, Müller S H, Reimold F, Stroth U, Wischmeier M, Wolfrum E, and the ASDEX Upgrade Team 2014 Nucl. Fusion 54123005

[4] Myra J R and D'Ippolito D A 2005 Phys. Plasmas 12092511

[5] Fuchert G, Birkenmeier G, Carralero D, Lunt T, Manz P, Müller H W, Nold B, Ramisch M, Rohde V, Stroth U, and the ASDEX Upgrade Team 2014 Plasma Phys. Control. Fusion 56 125001

[6] Boedo J A et al 2001 Phys. Plasmas 84826

[7] Nanobashvili I, Devynck P, Gunn J P, Nanobashvili S, Stöckel J, and Van Oost G 2009 Phys. Plasmas 16022309

[8] Nold B, Conway G D, Happel T, Müller H W, Ramisch M, Rohde V, Stroth U, and the ASDEX Upgrade Team 2010 Plasma Phys. Control. Fusion 52065005

[9] Happel T, Greiner F, Mahdizadeh N, Nold B, Ramisch M, and Stroth U 2009 Phys. Rev. Lett. 102255001

[10] Furno I, Labit B, Podestà M, Fasoli A, Müller S H, Poli F M, Ricci P, Theiler C, Brunner S, Diallo A, and Graves J 2008 Phys. Rev. Lett. 100055004

[11] Nold B, Ribeiro T T, Ramisch M, Huang Z, Müller H W, Scott B D, Stroth U, and the ASDEX Upgrade Team 2012 New J. Phys. 14063022

[12] Stroth U et al 2013 Nucl. Fusion 53104003

[13] Antar G Y 2003 Phys. Plasmas 103629

[14] Fuchert G, Birkenmeier G, Nold B, Ramisch M, and Stroth U 2013 Plasma Phys. Control. Fusion $\mathbf{5 5} 125002$

[15] Carralero D, Shoji M, de la Cal E, van Milligan B Ph, de Pablos J L, Hidalgo C, and Yamada H 2011 Contrib. Plasma Phys. 5192

[16] Carralero D, Calvo I, Shoji M, Carreras B A, Ida K, Ohdachi S, Sakakibara S, Yamada H, and Hidalgo C 2011 Plasma Phys. Control. Fusion 53095010

[17] Terry J L et al 2003 Phys. Plasmas 101739

[18] Maqueda R J, Stotler D P, Zweben S J 2011 J. Nucl. Mater. 415 S459

[19] Shesterikov I, Xu Y, Hidalgo C, Berte M, Dumontier P, Van Schoor M, Vergote M, and Van Oost G 2012 Nucl. Fusion 52042004

[20] Nold B, Manz P, Ribeiro T T, Fuchert G, Birkenmeier G, Müller H W, Ramisch M, Scott B D, and Stroth U 2014 Phys. Plasmas 21102304

[21] Manz P, Ribeiro T T, Scott B D, Birkenmeier G, Carralero D, Fuchert G, Müller S H, Müller H W, Stroth U, and Wolfrum E 2015 Phys. Plasmas 22022308

[22] Xu G S et al 2009 Nucl. Fusion 49092002

[23] Terry J L et al 2005 Nucl. Fusion 451321

[24] Madsen J, Garcia O E, Larsen J S, Naulin V, Nielsen A H, and Juul Rasmussen J 2011 Phys. Plasmas 18112504

[25] D'Ippolito D A and Myra J R 2003 Phys. Plasmas 104029

[26] Aydemir A Y 2005 Phys. Plasmas 12062503

[27] Stangeby P C 2000 The Plasma Boundary of Magnetic Fusion Devices, Taylor and Francis Group, New York

[28] Kočan M, Gennrich F P, Kendl A, Müller H W, and the ASDEX Upgrade Team 2012 Plasma Phys. Control. Fusion 54085009

[29] Angus J R, Umansky M V, and Krasheninnikov S I 2012 Phys. Rev. Lett. 108215002

[30] Walkden N R, Dudson B D, and Fishpool G 2013 Plasma Phys. Control. Fusion 55105005

[31] Zweben S J, Davis W M, Kaye S M, Myra J R, Bell R E, LeBlanc B P, Maqueda R J, Munsat T, Sabbagh S A, Sechrest Y, Stotler D P, and the NSTX Team 2015 Nucl. Fusion 55093035

[32] Fischer R, Fuchs C J, Kurzan B, Suttrop W, Wolfrum E, and the ASDEX Upgrade Team 2014 Fus. Sci. Techn. $\mathbf{5 8} 675$

[33] Garcia O E, Fritzner S M, Kube R, Cziegler I, LaBombard B, and Terry J L 2013 Phys. Plasmas 20055901 
[34] Birkenmeier G, Laggner F M, Willensdorfer M, Kobayashi T, Manz P, Wolfrum E, Carralero D, Fischer R, Sieglin B, Fuchert G, Stroth U, and the ASDEX Upgrade Team 2014 Plasma Phys. Control. Fusion 56075019

[35] Carralero D, Manz P, Aho-Mantila L, Birkenmeier G, Brix M, Groth M, Müller H W, Stroth U, Vianello N, Wolfrum E, ASDEX Upgrade team, JET Contributors, and EUROfusion MST1 Team 2015 Phys. Rev. Lett. 115215002

[36] Krasheninnikov S I 2001 Phys. Lett. A 283368

[37] Manz P, Carralero D, Birkenmeier G, Müller H W, Müller S H, Fuchert G, Scott B D, and Stroth U 2013 Phys. Plasmas 20102307

[38] Garcia O E, Bian N H, Naulin V, Nielsen A H, and Juul Rasmussen 2005 Phys. Plasmas 12 090701

[39] Myra J R, Russell D A, and D'Ippolito D A 2006 Phys. Plasmas 13112502

[40] Pitts R A et al 2011 J. Nucl. Mater 415 S957-S964

[41] Carralero D et al 2015 J. Nucl. Mater. 463 123-127

[42] Zohm H, Angioni C, Fable E et al 2013 Nucl. Fusion 53073019

[43] Lunt T, Carralero D, Feng Y, Birkenmeier G, Müller H W, Müller S, Wischmeier M, and the ASDEX Upgrade team $2015 \mathrm{~J}$. Nucl. Mater. 463 744-747

[44] LaBombard B, Boivin R L, Greenwald M, Hughes J, Lipschultz B, Mossessian D, Pitcher C S, Terry J L, Zweben S J, and Alcator Group 2001 Phys. Plasmas 82107

[45] Garcia O E, Horacek J, Pitts R A, Nielse A H, Fundamenski W, Naulin V, and Juul Rasmussen J 2007 Nucl. Fusion 47667

[46] Garcia O E 2012 Phys. Rev. Lett. 108265001 